\title{
Visual cortical simple cells: Who inhibits whom
}

\author{
ALAN B. SAUL \\ Department of Neurobiology, University of Pittsburgh School of Medicine, Pittsburgh \\ (Received October 1, 1998; AcCePTED January 25, 1999)
}

\begin{abstract}
Simple cells display a specific adaptation aftereffect when tested with drifting gratings. The onset of the response to each cycle of the grating is delayed after adapting, but the offset is unaffected. Testing with stationary bars whose luminance was modulated in time revealed that aftereffects occur only at certain points in both space and time. The aftereffects seen with moving stimuli were predicted from those seen with stationary stimuli. These adaptation experiments suggest a model that consists of mutually inhibitory simple cells that are in spatiotemporal quadrature. The inhibition is appropriately localized in space and time to create the observed aftereffects. In this model, inhibition onto direction-selective simple cells arises from simple cells with the same preferred direction.
\end{abstract}

Keywords: Spatiotemporal quadrature, Adaptation aftereffects, Response timing, Direction selectivity, Negative feedback

\section{Introduction}

Adaptation aftereffects in single cells of cat visual cortex appear in the timing as well as in the strength of their responses. A highly specific timing aftereffect is seen when simple cells are presented with drifting gratings, consisting of a delay in the onset of response to each cycle of the test stimulus but no change in the offset (Saul, 1995). A model presented here accounts for the previous as well as new experimental results.

This model also addresses how inhibition is tuned for direction. A common view of the creation of cortical selectivity involves convergence of excitatory inputs that support responses to optimal stimuli, combined with inhibitory influences from cortical cells with opposing stimulus preferences. In the visual system, orientation selectivity is often thought to be generated by excitatory input from cells whose receptive fields are aligned along the preferred orientation (Hubel \& Wiesel, 1962), with inhibition from cells preferring different orientations (Sillito et al., 1980; Ferster \& Koch, 1987). Direction selectivity has been modeled by analogous mechanisms (Sillito, 1984; Maex \& Orban, 1995).

This idea that inhibition is tuned in a way that opposes the excitation is not well supported experimentally (Creutzfeldt et al., 1974; Innocenti \& Fiore, 1974; Ferster, 1988; Sato et al., 1991; Murthy \& Humphrey, 1999). Instead, the tuning of inhibition tends to match that of the excitation. The model outlined here shows how to make sense of inhibition between simple cells with similar direction preference.

Correspondence and reprint requests to: Alan B. Saul, Department of Neurobiology, University of Pittsburgh School of Medicine, Pittsburgh, PA 15261, USA. E-mail: saul+@ @itt.edu

\section{Methods}

Details of the experimental design can be found elsewhere (Saul, 1995). Briefly, female adult cats were anesthetized with halothane, immobilized with Flaxedil and curare, and artificially respired. All procedures were performed according to the NIH Guidelines for the care and use of animals. Blood pressure, heart rate, and EEG were monitored for signs of discomfort.

Extracellular single-unit recordings were made from simple cells in area 17. Visual stimuli were presented at a mean luminance of $15 \mathrm{~cd} / \mathrm{m}^{2}$. Either a blank screen or a drifting grating was present during the first $8 \mathrm{~s}$ of each 12-s trial. The subsequent $4 \mathrm{~s}$ were used to collect data in response to a test stimulus, which was either a drifting grating or a stationary bar. From trial to trial, the bar was placed at a series of positions across the receptive field and its luminance was modulated sinusoidally in time at a series of temporal frequencies (Saul \& Humphrey, 1992). Comparisons were made between responses to the identical test stimulus under two conditions: control and adapted. Control responses were those that followed $8 \mathrm{~s}$ of the blank screen; adapted responses were those that followed $8 \mathrm{~s}$ of the drifting grating. The various conditions were interleaved in pseudorandom sequences.

Responses to the stationary bars were used to predict the responses to the drifting grating. The prediction was based on the relation

$$
R(t)=\int r(x, t-x f / \omega) \mathrm{d} x,
$$

where $R(t)$ is the predicted response to a grating of spatial frequency $f$ drifting at temporal frequency $\omega$, and $r(x, t)$ is the response to a stationary bar. Bars were presented at a series of positions $x$, and the integral was approximated as a sum over the 
tested positions. Predictions were computed for spatial frequencies between $\pm 1 /(2 \Delta x)$, where $\Delta x$ is the distance between adjacent positions. Predicted responses shown here were derived from the spatial frequency giving the largest first harmonic amplitude for the control case.

Model responses were computed by an iterative method that relaxed to a stable state, to simulate the feedback between two mutually inhibitory simple cells. The excitatory afferent inputs to the simple cells were rectified products of spatial and temporal sinusoids weighted by a Gaussian function of space (Fig. 4B). The gain of the inhibition between the simple cells was the only parameter that changed across simulations, increasing in the adapted state relative to the control state (Fig. 5). The inhibition was lowpass filtered by convolving the cortical activity with an exponential decay whose time constant was an eighth of a cycle, creating a slight delay of the postsynaptic effect relative to the presynaptic activity. On each iteration: (1) the inhibition was computed by multiplying the low-passed cortical activity by the gain; (2) the inhibition was subtracted from the afferent excitation; and (3) the cortical responses were rectified. Step 3 is the only nonlinearity in this process. Thus, the response of one of the cells at each iteration was a function of the response of the other cell at the previous iteration. The sequence was considered to have converged when the absolute difference between responses in successive iterations at all positions and times was less than $0.1 \%$ of the peak response. The process is given by the equations:

$$
\begin{aligned}
& A_{0}=E_{A}, \\
& B_{0}=E_{B}, \\
& A_{n}=R\left(E_{A}-q \cdot B_{n-1} * F\right), \\
& B_{n}=R\left(E_{B}-q \cdot A_{n-1} * F\right),
\end{aligned}
$$

where $A_{n}$ and $B_{n}$ are the responses of the two cells at the $n$th iteration, $R(u)=\max (0, u)$ is rectification, $E_{A}$ and $E_{B}$ are the afferent inputs, $q$ is the inhibitory gain, $*$ indicates convolution, and $F$ is the low-pass filter.

\section{Results}

\section{Experimental}

The timing aftereffect is illustrated in Fig. 1A. The control response was obtained from a 0.5 contrast grating drifting across the simple cell's receptive field at $2 \mathrm{~Hz}$ for $4 \mathrm{~s}$. The adapted response was obtained from the identical test stimulus, but was preceded by $8 \mathrm{~s}$ of the same 0.5 contrast grating, whereas a blank screen preceded the interleaved test trials from which the control responses were recorded.

The onset of the adapted response was delayed at each cycle of the stimulus ( 8 cycles here during the 4 -s test trials). However, the cell responded equally well under both conditions later in the cycle, and the offset of the response was not affected. To explain this result, some nonlinear mechanisms are required, since any linear transformation of the control response will shift both onset and offset by the same amount. However, a quasilinear model can reproduce this behavior (Saul, 1995). This model consists of mutually inhibitory simple cells that are in a push-pull relationship. The only nonlinearity required is rectification.
On the other hand, stronger nonlinearities could conceivably explain the result. One way to test this is to use the fact that responses to moving stimuli in many simple cells have a quasilinear relationship to responses to stationary stimuli. If, for example, nonlinearities inherent to moving stimuli were involved in these aftereffects, they would not be seen in the stationary tests.

Receptive fields were therefore mapped with stationary stimuli in control and adapted states, and the responses to moving stimuli were computed through linear predictions based on the stationary responses. As described previously (Saul \& Humphrey, 1992), maps were derived from responses to sinusoidally luminancemodulated stationary bars. In Fig. 1B, the control and adapted maps are shown for $2-\mathrm{Hz}$ modulation. Three positions were still driven well in the adapted condition, two ON positions (at $0.4 \mathrm{deg}$ and $0.1 \mathrm{deg}$ ) and one OFF position (at $-0.1 \mathrm{deg}$ ). Two of these positions were not affected by adapting, but at $0.4 \mathrm{deg}$ a timing aftereffect like those seen with moving stimuli is apparent. All adapting stimuli here were moving gratings, which might be expected to affect all positions across the receptive field similarly. The fact that aftereffects occur at specific points in space and time provides a powerful challenge to models. Predictions of the responses to moving stimuli are shown in Fig. 1C, and display a timing aftereffect, indicating that the aftereffect seen with moving stimuli does not necessarily involve processes beyond those present in the receptive-field structure.

Fig. 2 illustrates analogous results from three additional neurons. These cells illustrate the wide range of detailed behaviors observed in a small sample. The only general conclusion was that all 12 simple cells that responded adequately in the adapted state (adapting often simply eliminated responsiveness) showed timing aftereffects at some but not all positions. In Fig. 2A, a cell shows only minimal timing aftereffects in its $\mathrm{ON}$ zone. The cell in Fig. 2B exhibited timing aftereffects in its OFF zone and at one position in its $\mathrm{ON}$ zone. The cell in Fig. 2C displayed strong aftereffects at several positions. Fig. 3 shows a cell responding in control and adapted states at three temporal frequencies. Aftereffects appeared at several positions at each of the tested temporal frequencies, but these positions changed with frequency. No conclusions about how the affected positions related to receptive-field structure and direction selectivity could be drawn from these limited experiments. The model presented below hypothesizes that stimulation at these positions evoked appropriately timed inhibition.

\section{Theoretical}

The highly reduced model (Fig. 4A) consists of two mutually inhibitory simple cells that receive excitatory lagged or nonlagged lateral geniculate nucleus (LGN) inputs. The separation of the lagged and nonlagged projections here was chosen to clarify the model, not to match any of the particular examples in Figs. 1-3. Each cell could have been shown as receiving convergent lagged and nonlagged inputs, but presenting the model without this convergence emphasizes the intracortical interaction. The $\mathrm{ON}$ - and OFF-center LGN cells have spatially offset receptive fields. The spatial relationships between the lagged and nonlagged LGN cells are important: the receptive fields of the lagged and nonlagged cells interdigitate (Saul \& Humphrey, 1992). The ON and OFF zones of each simple cell lie a half-cycle apart in space, and the lagged and nonlagged zones are a quarter-cycle apart in space. Lagged and nonlagged LGN cells respond about a quarter-cycle apart in time (Saul \& Humphrey, 1990). The result is that the LGN inputs provide "spatiotemporal quadrature." The key aspect of the 
model is that the mutually inhibitory simple cells respond at different points in space and in time.

Fig. 4B presents space-time plots that show how the components of this model respond to sinusoidally modulated stationary stimuli like those used in the experiment of Fig. 1B. The afferent activity is indicated above the model cortical responses. The inhibitory relays provide spatiotemporal quadrature partners of the excitatory afferents. Comparing the upper to the lower plots in Fig. 4B, one can see that the afferent excitation is suppressed at some receptive-field positions and times but not at others, depending on the responses providing the inhibition. Because the excitation and the inhibition are offset in space and time, their interaction produces the spatiotemporal orientation of the simple cell responses, seen as the upper right to lower left slope of the cortical maps.
A

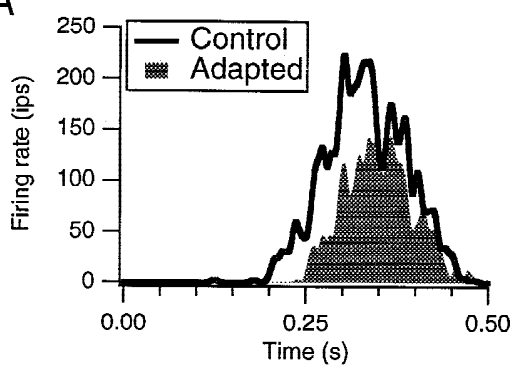

$\mathrm{B}$
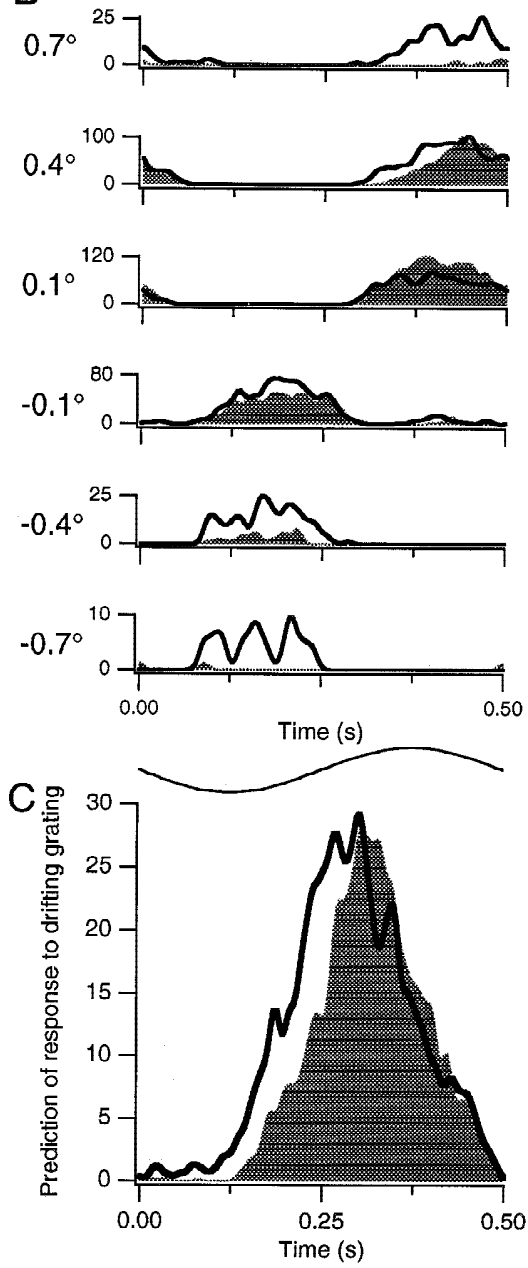

Spatiotemporally oriented receptive fields are direction selective, with the preferred direction being that in which the responses occur progressively earlier in time. Note that both receptive fields are oriented in the same direction, preferring motion downward through their receptive fields. The counterintuitive notion that cells that inhibit each other prefer the same direction of motion is not surprising here, since both these cells receive the same inputs, with only a reversal of sign. The receptive fields are not separated by a half-cycle, however. Instead, they differ by a quarter-cycle in space and a quarter-cycle in time.

Simulated responses to drifting gratings are illustrated in Fig. 4C. The two model neurons respond out of phase with each other in their preferred direction, silencing each other during alternate half-cycles. Even though the strongest inhibition arrives onto each cell for the preferred direction, this inhibition does not reduce the response, because it occurs in time when there is little excitation. In the nonpreferred direction, on the other hand, the quarter-cycle differences between the cells cancel each other, and the two cells are active at the same time. They inhibit each other and reduce each other's response in this direction.

The inhibition in the nonpreferred direction creates the direction selectivity. Nonetheless the amplitude of these inhibitory signals is low, since the presynaptic cell is not as active as in the preferred direction. The apparent contradiction that inhibition is crucial yet weak arises from the way negative feedback regulates activity. The strength of inhibition is directly, rather than inversely, related to the strength of excitatory responses.

The model of Fig. 4 can reproduce the adaptation data described above. The responses of the model cells derived in Fig. 4 will be considered to be the control responses. To obtain the adapted responses, the inhibitory synapses are potentiated (their gain is increased). Results for moving stimuli have been previously described (Saul, 1995).

In Fig. 5A, the stationary responses are similar to those in Fig. $1 \mathrm{~B}$ in that some positions are not affected by adapting, but others show a delayed onset with no change in offset. In Fig. 5B, the moving responses have been predicted from the stationary responses in Fig. 5A, for comparison to Fig. 1C. The onset is delayed, with no change in offset. Timing aftereffects can be explained by quasilinear mechanisms if response timing is taken into account.

Fig. 1. Example of timing aftereffects from a direction-selective (DS = 0.81 ) simple cell in layer 4B. A: Extracellularly recorded responses of a simple cell to a grating drifting across its receptive field in the preferred direction in control and adapted states. Both responses are to the same stimulus, a 0.5 contrast 0.7 -cpd grating moving at $2 \mathrm{~Hz}$ for $4 \mathrm{~s}$, so that the histograms are averages over 8 cycles and 5 repeated trials (40 cycles total). The control response was obtained in trials that followed $8 \mathrm{~s}$ of a blank screen, whereas the adapted response came from interleaved trials that followed $8 \mathrm{~s}$ of the same grating as used for the test responses. Thus, one can think of these responses as arising from the first $4 \mathrm{~s}$ or the last $4 \mathrm{~s}$ of a 12-s presentation of the grating. B: Responses of a simple cell to sinusoidally modulated stationary bars in control and adapted states. All histograms have been smoothed. Bars were $0.2 \mathrm{deg} \times 4 \mathrm{deg}$, presented in 4-s trials following $8 \mathrm{~s}$ of either a blank screen or a drifting grating as in A. Note that the vertical scale for firing rate (in impulses/s) varies across the six positions shown and that the responses at $-0.1 \mathrm{deg}, 0.1 \mathrm{deg}$, and 0.4 deg dominate. C: Predictions of responses to drifting gratings based on actual responses to stationary bars shown in B. Data were summed after shifting in time appropriately for a 0.7 -cpd grating moving downward through the receptive field. 
A

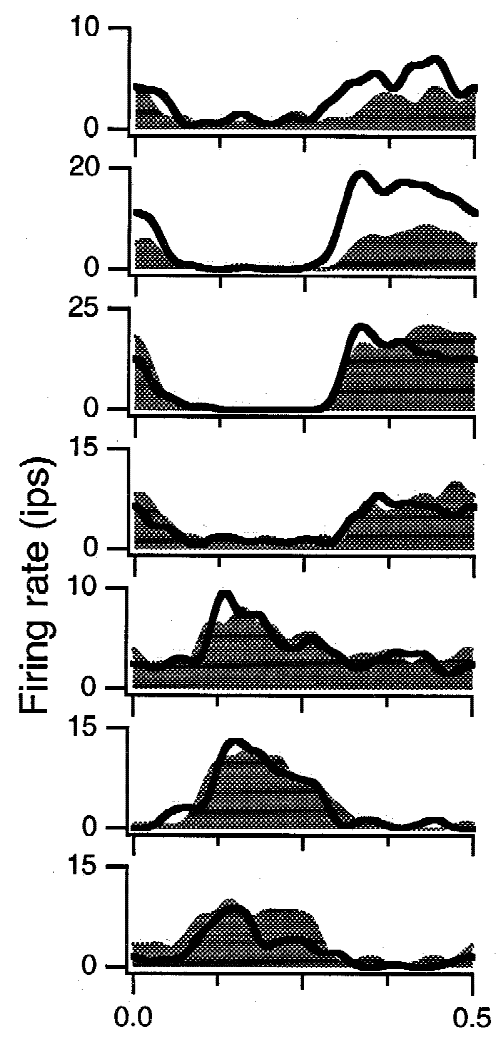

B
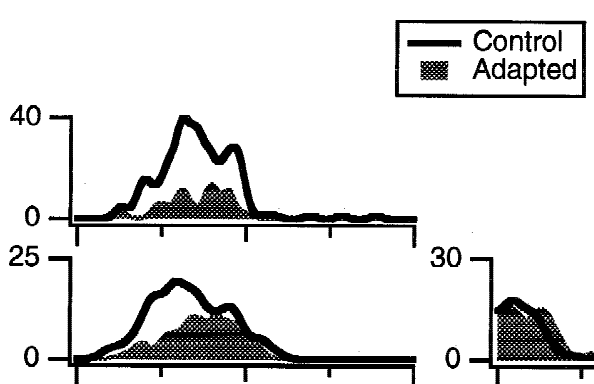

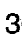
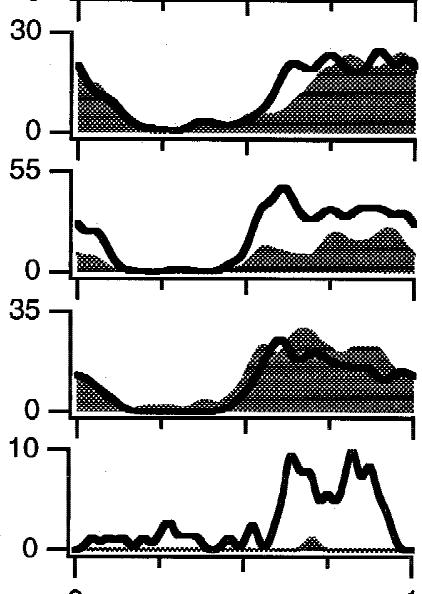

0
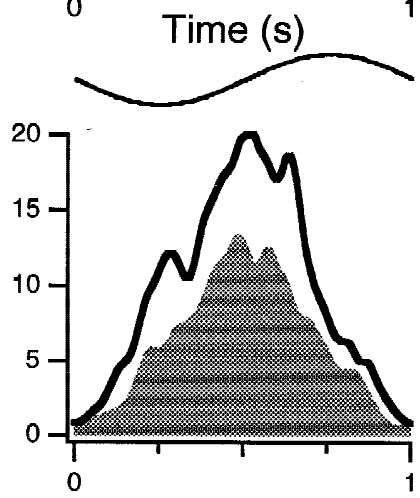

C
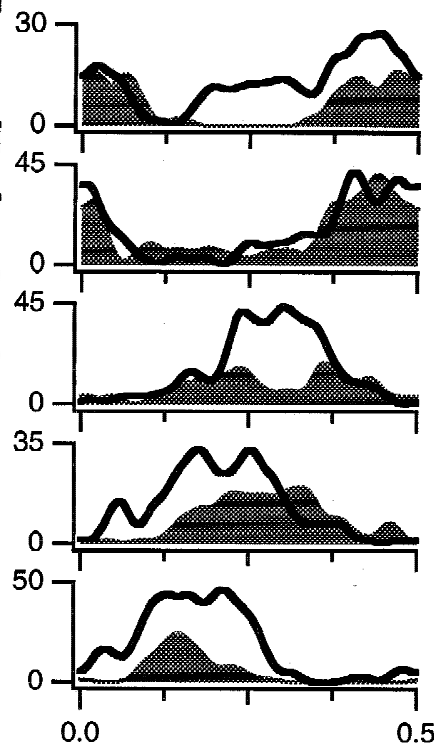

0.5

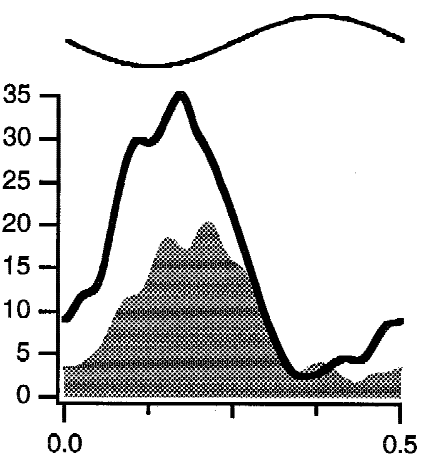

Fig. 2. Three examples of aftereffects in spatiotemporal receptive-field maps. Format is like that of Fig. 1B, with predictions as in Fig. 1C shown below each series of positions. Positions where responses were unreliable (standard error of phase exceeded 0.1 cycle or standard error of amplitude exceeded half mean amplitude) were omitted for clarity here and in Figs. 1 and 3. A: Responses at $2 \mathrm{~Hz}$ from a cell in layer 6 that was not direction selective $(\mathrm{DS}=0.26$ ). Positions shown ranged over $1.7 \mathrm{deg}$. Test bar was $0.2 \mathrm{deg} \times 8 \mathrm{deg}$. Adapting grating had a temporal frequency of $2 \mathrm{~Hz}$ and a spatial frequency of $0.4 \mathrm{cpd}$. Contrast was $0.5 \mathrm{for}$ all stimuli. Spatial frequency used for the prediction was $0.37 \mathrm{cpd}$. B: Responses over $1.5 \mathrm{deg}$ at $1 \mathrm{~Hz}$ from a moderately direction selective (DS $=0.62)$ layer $4 \mathrm{~A}$ cell. Parameters of adapting grating were 0.5 contrast, $1 \mathrm{~Hz}, 0.8 \mathrm{cpd}$. Test contrast was 0.4 , bar was $0.3 \mathrm{deg} \times 8 \mathrm{deg}$. Prediction was obtained at $0.8 \mathrm{cpd}$. C: Responses over $0.6 \mathrm{deg}$ at $2 \mathrm{~Hz}$ from a direction-selective ( $\mathrm{DS}=0.72$ ) cell in layer 4B. Adapting grating was 0.5 contrast, $2 \mathrm{~Hz}, 1.6 \mathrm{cpd}$. Test bar was 0.8 contrast, $0.2 \mathrm{deg} \times 4 \mathrm{deg}$. Prediction was obtained at $1.01 \mathrm{cpd}$.

\section{Discussion}

Direction selectivity emerges through spatial and temporal differences between inputs. Potential sources of the temporal differences underlying cortical direction selectivity in the cat are lagged and nonlagged cells in the lateral geniculate nucleus (Saul \& Humphrey, 1990). Cortical cells can therefore receive excitatory afferents that are in approximate spatiotemporal quadrature. The combined geniculate inputs to single cortical simple cells can be direction selective (Ferster et al., 1996; Chung \& Ferster, 1998). Nonetheless, cortical cells excite and inhibit each other, and in the process relay the signals obtained directly from the afferents. Intracortical excitation was not considered explicitly here, since its tuning is more straightforward (Maex \& Orban, 1995; Suarez et al., 1995) than that of inhibition. Intuitively, and according to some models (Sillito, 1984; Maex \& Orban, 1995), inhibition should be strongest in the nonpreferred direction. 


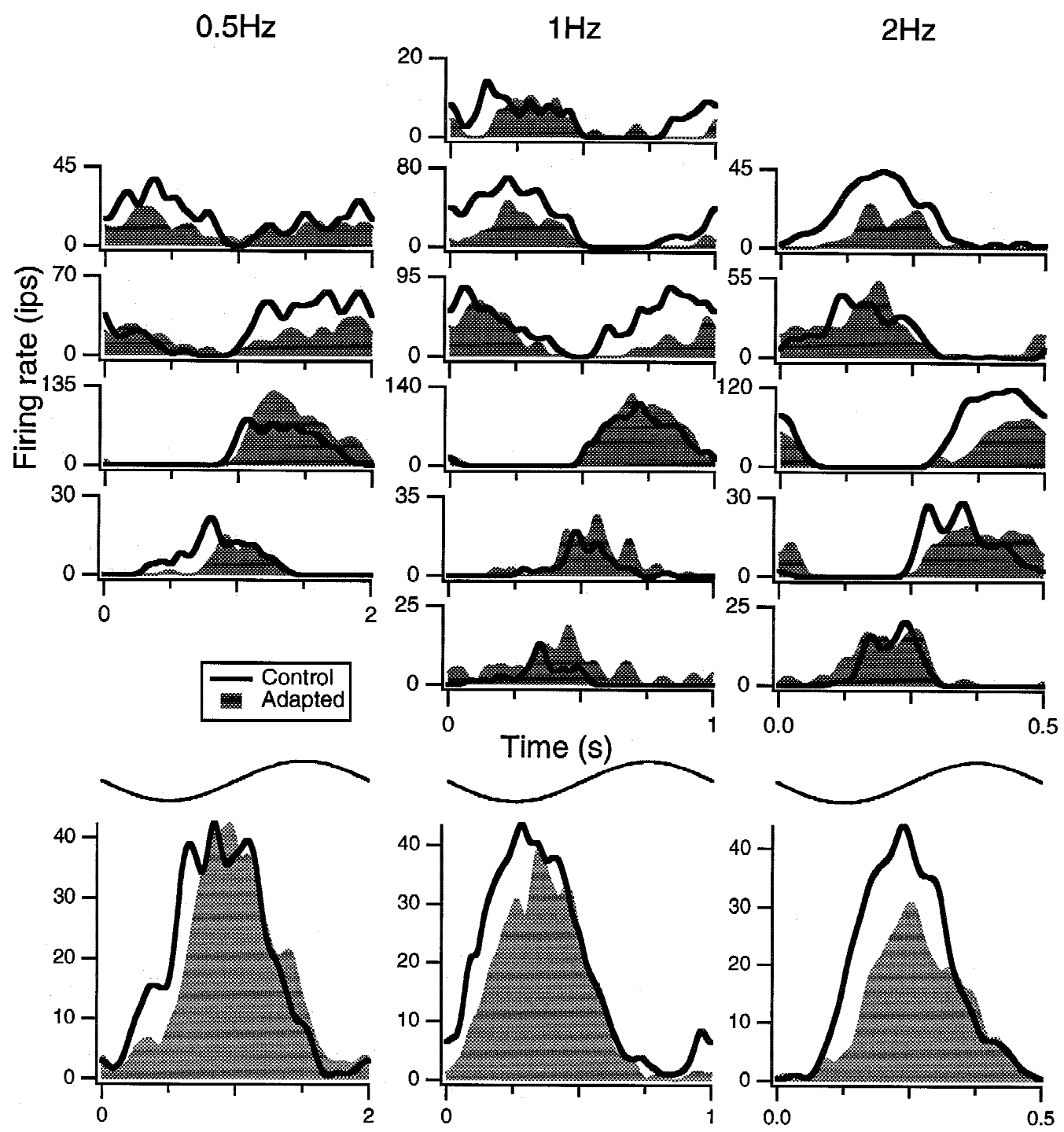

Fig. 3. Aftereffects at three test temporal frequencies in a direction-selective ( $\mathrm{DS}=0.96)$ simple cell in mid-layer 4 . Adapting stimulus was a $1-\mathrm{Hz} 0.6-\mathrm{cpd}$ grating. Test bar was $0.2 \mathrm{deg} \times 8 \mathrm{deg}$. All stimuli were at 0.5 contrast. Positions shown ranged over $1.5 \mathrm{deg}$ (at $1 \mathrm{~Hz}$ ). Predictions were obtained at $0.7 \mathrm{cpd}$.

Adaptation was assumed here to potentiate inhibition. This assumption is not supported by pharmacological experiments that blocked GABA receptors (DeBruyn \& Bonds, 1986; Vidyasagar, 1990; McLean \& Palmer, 1996). Those studies only examined amplitude aftereffects. Antagonizing $\mathrm{GABA}_{\mathrm{A}}$-mediated inhibition could interfere with timing aftereffects, since aftereffects of timing and amplitude are independent (Saul, 1995). A portion of that independence can be attributed to "intrinsic," fatigue-like mechanisms underlying some amplitude aftereffects (Vautin \& Berkley, 1977). For instance, a nonspecific intrinsic decrease in response amplitude at low contrasts is probably mediated by a prominent membrane hyperpolarization (Carandini \& Ferster, 1997; SanchezVives et al., 1997) that seems to be independent of inhibitory inputs. These changes in amplitude may or may not be accompanied by changes in timing. Adapting often reduced response amplitude to zero at some positions in the present experiments, making it impossible to measure timing (these positions were generally omitted from Figs. 1-3). However, it is clear that timing can change without any change in amplitude. In many cases, timing aftereffects are observed at high contrasts without a large decrease in amplitude (Saul, 1995), consistent with the high-contrast data shown by Carandini and Ferster (1997). Amplitude aftereffects are reduced by antagonism of presynaptic metabotropic glutamate receptors (McLean \& Palmer, 1996). It remains unclear how this or other mechanisms for synaptic depression could mimic the observed timing aftereffects, whereas the model relying on inhibition gives an excellent fit to the data.

This model, which extends ideas of many others (Hubel \& Wiesel, 1959; Palmer \& Davis, 1981; Ferster, 1986; Heggelund, 1986; Ferster \& Koch, 1987; Tolhurst \& Dean, 1987, 1990; McLean et al., 1994; Livingstone, 1998), calls attention to the relative spatiotemporal organization of the net excitatory and inhibitory input to a cell. For direction-selective cells, the excitation and inhibition are in approximate quadrature. Most previous studies have not focused on the temporal differences between excitation and inhibition, often assuming that they are a half-cycle out of phase. The 
A

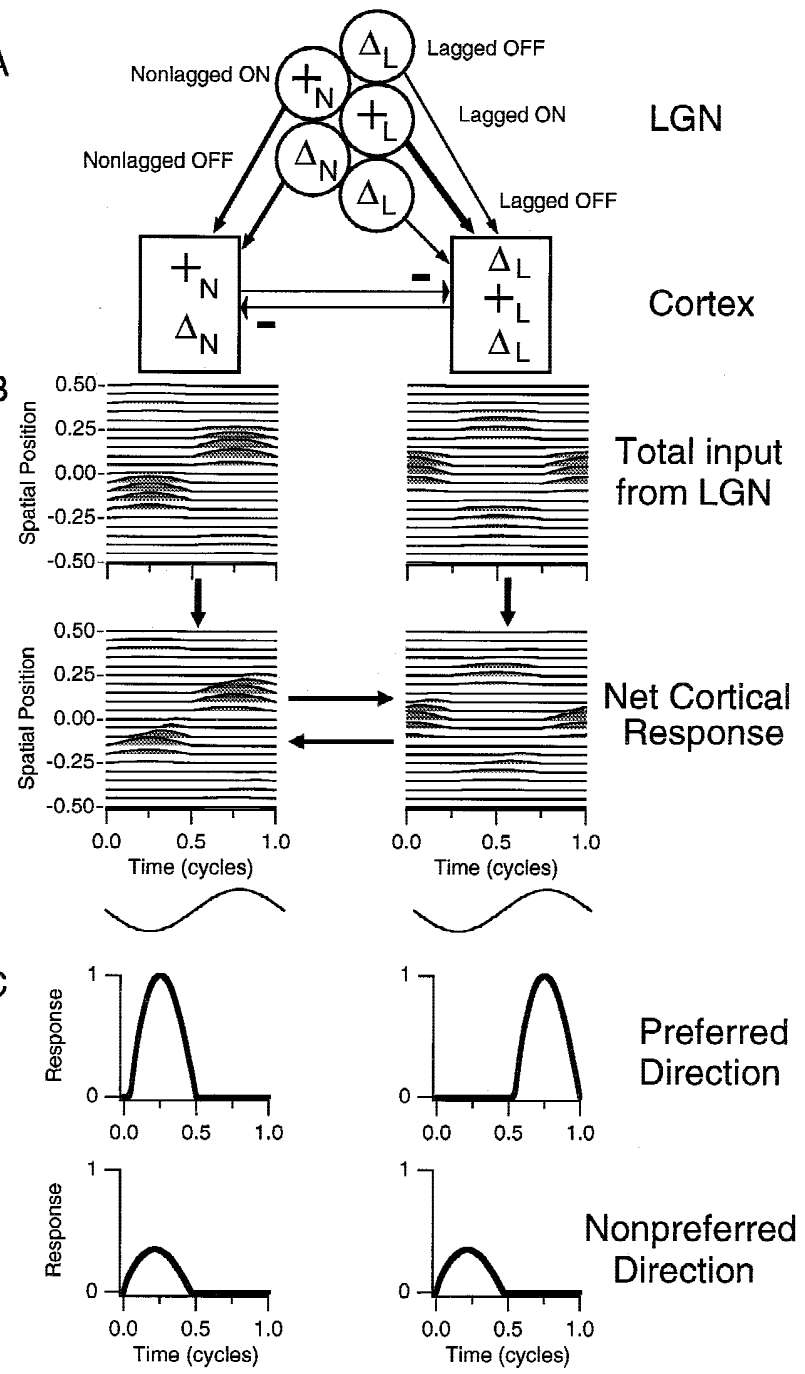

Fig. 4. Model of mutually inhibitory simple cells. A: ON and OFF center lagged and nonlagged inputs from the LGN project to two cortical cells that inhibit each other. The simple cell on the left receives ON and OFF nonlagged inputs, while the simple cell on the right receives input from $\mathrm{ON}$ and OFF lagged LGN cells. Only one spatial dimension is represented here; the horizontal offset between the lagged and nonlagged cells is for clarity. B: Space-time receptive-field maps of the inputs and of the simple cells. These maps can be interpreted as responses to sinusoidally modulated stationary bars. The inputs to the cell on the left are centered at \pm 0.17 and occur at the luminance trough or peak $(\sin 3 \pi x \cdot \sin 2 \pi t)$. The inputs to the other cell are shifted in space and in time to fall in between the left-hand inputs $(\cos 3 \pi x \cdot \cos 2 \pi t)$. All responses are rectified. The amplitude of these responses is modulated by a Gaussian in space to localize the receptive field. The cortical responses show the effects of the mutual inhibition, which reduces responses at certain positions and times. These responses were computed by an iterative method that simulates the feedback. C: Simulated responses of the cortical cells to a grating drifting in each direction (preferred is downward in B and nonpreferred is upward) across the receptive field. These are not the results of integrating the histograms in B (as in Figs. 1C or 5B) but instead are comparable to Fig. 1A, modeling the actual responses to motion: sinusoidal functions of time were provided as the excitatory input to the model cortical cells, ignoring any explicit spatial dependence.
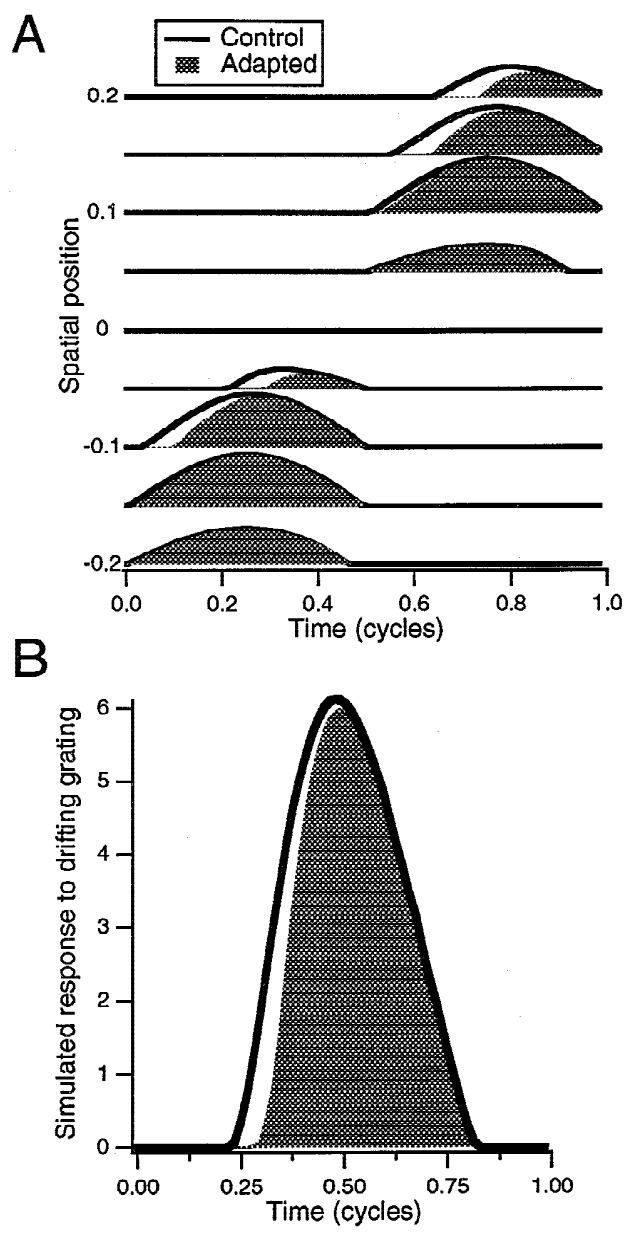

Fig. 5. Simulation of model responses to stationary and drifting stimuli in control and adapted states. The only difference between these states is in the strength of the inhibitory connections. A: These histograms represent the same model responses as shown for the simple cell on the left in Fig. $4 \mathrm{~B}$, with the adapted responses taken after increasing the gain of the mutual inhibition by a factor of 5. B: Predicted responses to a drifting grating based on the responses in A.

claim here is that the relative phase of the excitatory and inhibitory inputs varies from cell to cell. These inputs are ultimately derived from the LGN, but can be relayed through other cortical cells. The inhibitory relays connect cells that prefer the same direction but respond at different times in that direction. This scheme accounts for the adaptation data, which present a challenge to models for cortical receptive-field structure and direction selectivity.

\section{Acknowledgments}

This research was supported by NSF Grant BNS 9021495, NIH EY10826, and a Core Grant for Vision Research (EY08098) to the Eye and Ear Institute of Pittsburgh. I am grateful to A. Humphrey, A. Murthy, J. Feidler, E. Frank, B. Minnery, and P. Baker for their assistance.

\section{References}

Carandini, M. \& Ferster, D. (1997). A tonic hyperpolarization underlying contrast adaptation in cat visual cortex. Science 276, 949-952.

Chung, S. \& Ferster, D. (1998). Strength and orientation tuning of the thalamic input to simple cells revealed by electrically evoked cortical suppression. Neuron 20, 1177-1189. 
Creutzfeldt, O.D., Kuhnt, U. \& Benevento, L.A. (1974). An intracellular analysis of visual cortical neurons to moving stimuli: Responses in a co-operative neuronal network. Experimental Brain Research 21, 251-274.

DeBruyn, E.J. \& Bonds, A.B. (1986). Contrast adaptation in cat visual cortex is not mediated by GABA. Brain Research 383, 339-342.

Ferster, D. (1986). Orientation selectivity of synaptic potentials in neurons of cat visual cortex. Journal of Neuroscience 6, 1284-1301.

Ferster, D. \& KосH, C. (1987). Neuronal connections underlying orientation selectivity in cat visual cortex. Trends in Neuroscience 10, 487492.

FERSTER, D. (1988). Spatially opponent excitation and inhibition in simple cells of the cat visual cortex. Journal of Neuroscience 8, 1172-1180.

Ferster, D., Chung, S. \& Wheat, H. (1996). Orientation selectivity of thalamic input to simple cells of cat visual cortex. Nature 380, 249252.

Heggelund, P. (1986). Quantitative studies of enhancement and suppression zones in the receptive field of simple cells in cat striate cortex. Journal of Physiology 373, 293-310.

Hubel, D.H. \& Wiesel, T.N. (1959). Receptive fields of single neurones in cat's visual cortex. Journal of Physiology 148, 574-591.

Hubel, D.H. \& Wiesel, T.N. (1962). Receptive fields, binocular interaction, and functional architecture in the cat's visual cortex. Journal of Physiology 160, 106-154.

InNOCEnTi, G.M. \& Fiore, L. (1974). Post-synaptic inhibitory components of the responses to moving stimuli in area 17. Brain Research 80, $122-126$.

Livingstone, M.S. (1998). Mechanisms of direction selectivity in macaque V1. Neuron 20, 509-526.

Maex, R. \& Orban, G. (1995). Model circuit of spiking neurons generating directional selectivity in simple cells. Journal of Neurophysiology 75, 1515-1545

McLean, J. \& Palmer, L.A. (1996). Contrast adaptation and excitatory amino acid receptors in cat striate cortex. Visual Neuroscience 13, 1069-1087.

Mclean, J., RaAb, S. \& Palmer, L.A. (1994). Contribution of linear mechanisms to the specification of local motion by simple cells in areas 17 and 18 of the cat. Visual Neuroscience 11, 271-294.

Murthy, A. \& Humphrey, A.L. (1999). Inhibitory contributions to spa- tiotemporal receptive-field structure and direction selectivity in simple cells of cat area 17. Journal of Neurophysiology 81, 1212-1224.

Palmer, L.A. \& DAVIS, T.L. (1981). Receptive-field structure in cat striate cortex. Journal of Neurophysiology 46, 260-276.

Sanchez-Vives, M.V., NowaK, L.G. \& McCormick, D.A. (1997). Cellular and network mechanisms generating adaptation to contrast in the visual cortex: An in vivo and in vitro study. Society for Neuroscience Abstracts 23, 1944.

Sato, H., Daw, N.W. \& Fox, K. (1991). An intracellular recording study of stimulus-specific response properties in cat area 17. Brain Research 544, 156-161.

Saul, A.B. \& Humphrey, A.L. (1990). Spatial and temporal response properties of lagged and nonlagged cells in cat lateral geniculate nucleus. Journal of Neurophysiology 64, 206-224.

Saul, A.B. \& Humphrey, A.L. (1992). Evidence of input from lagged cells in the lateral geniculate nucleus to simple cells in cortical area 17 of the cat. Journal of Neurophysiology 68, 1190-1208.

SAUL, A.B. (1995). Adaptation aftereffects in single neurons of cat visual cortex: Response timing is retarded by adapting. Visual Neuroscience 12, 191-205.

Sillito, A.M., Kemp, J.A., Milson, J.A. \& Berardi, N. (1980). A reevaluation of the mechanisms underlying simple cell orientation selectivity. Brain Research 194, 517-520.

Sillito, A.M. (1984). Functional considerations of the operation of GABAergic inhibitory processes in the visual cortex. In Cerebral Cortex, Vol. 2, ed. Jones, E.G. \& Peters, A., pp. 91-117. New York: Plenum Press.

Suarez, H., Koch, C. \& Douglas, R. (1995). Modeling direction selectivity of simple cells in striate visual cortex using the canonical microcircuit. Journal of Neuroscience 15, 6700-6719.

Tolhurst, D. J. \& Dean, A. F. (1987). Spatial summation by simple cells in the striate cortex of the cat. Experimental Brain Research 66, 607-620.

Tolhurst, D.J. \& Dean, A.F. (1990). The effects of contrast on the linearity of spatial summation of simple cells in the cat's striate cortex. Experimental Brain Research 79, 582-588.

VAutin, R.G. \& BerKLEY, M.A. (1977). Responses of single cells in cat visual cortex to prolonged stimulus movement: Neural correlates of visual after effects. Journal of Neurophysiology 40, 1051-1065.

Vidyasagar, T.R. (1990). Pattern adaptation in cat visual cortex is a cooperative phenomenon. Neuroscience 36, 175-179. 